\title{
Clinical Decision Support Functions and Digitalization of Clinical Documents of Electronic Medical Record Systems
}

\author{
Young-Taek Park ${ }^{1}$, Yeon Sook Kim² ${ }^{2}$, Byoung-Kee Yi ${ }^{3,4}$, Sang Mi Kim ${ }^{5}$ \\ ${ }^{1}$ Research Institute for Health Insurance Claims Review \&t Assessment, Health Insurance Review \& Assessment Service, Wonju, Korea \\ ${ }^{2}$ Department of Nursing, California State University, San Bernardino, CA, USA \\ ${ }^{3}$ Smart Healthcare \& Device Research Center, Samsung Medical Center, Seoul, Korea \\ ${ }^{4}$ Samsung Advanced Institute of Health Science and Technology, Sungkyunkwan University, Seoul, Korea \\ ${ }^{5}$ Department of Big Data Analytics, Ewha Womans University, Seoul, Korea
}

Objectives: The objective of this study was to investigate the clinical decision support (CDS) functions and digitalization of clinical documents of Electronic Medical Record (EMR) systems in Korea. This exploratory study was conducted focusing on current status of EMR systems. Methods: This study used a nationwide survey on EMR systems conducted from July 25, 2018 to September 30, 2018 in Korea. The unit of analysis was hospitals. Respondents of the survey were mainly medical recorders or staff members in departments of health insurance claims or information technology. This study analyzed data acquired from 132 hospitals that participated in the survey. Results: This study found that approximately $80 \%$ of clinical documents were digitalized in both general and small hospitals. The percentages of general and small hospitals with $100 \%$ paperless medical charts were $33.7 \%$ and $38.2 \%$, respectively. The EMR systems of general hospitals are more likely to have CDS functions of warnings regarding drug dosage, reminders of clinical schedules, and clinical guidelines compared to those of small hospitals; this difference was statistically significant. For the lists of digitalized clinical documents, almost 93\% of EMR systems in general hospitals have the inpatient progress note, operation records, and discharge summary notes digitalized. Conclusions: EMRs are becoming increasingly important. This study found that the functions and digital documentation of EMR systems still have a large gap, which should be improved and made more sophisticated. We hope that the results of this study will contribute to the development of more sophisticated EMR systems.

Keywords: Electronic Medical Records, Electronic Health Records, Computerized Medical Record Systems, Medical Informatics, Health Information Exchange

Submitted: March 28, 2019

Revised: April 21, 2019

Accepted: April 22, 2019

\section{Corresponding Author}

Young-Taek Park

Health Insurance Review \& Assessment Service, 60, Hyeoksin-ro, Wonju 26465, Korea. Tel: +82-33-739-0944, E-mail: pyt0601@hira.or.kr (https://orcid.org/0000-0002-7574-4165)

This is an Open Access article distributed under the terms of the Creative Commons Attribution Non-Commercial License (http://creativecommons.org/licenses/by-nc/4.0/) which permits unrestricted non-commercial use, distribution, and reproduction in any medium, provided the original work is properly cited.

(C) 2019 The Korean Society of Medical Informatics 


\section{Introduction}

Electronic Medical Record/Electronic Health Record (hereafter, EMR) systems have been widely adopted and used in most healthcare organizations [1-3]. The EMR is defined as a digital version of legal medical records or charts in clinical settings [4]. Thus, EMR systems could be descriptively defined as computer systems dealing with EMRs. It is important to know to what degree medical charts are digitalized and what functionalities EMR systems have to maximally use EMR systems in clinical settings.

EMR systems have a wide variety of functionalities for healthcare professionals to manage various health problems of patients $[1,5]$. Examples of these functionalities are healthcare information exchange (HIE) and tools or functions of clinical decision support (CDS). CDS could be defined as encompassing various tools of computer systems to enhance a healthcare professional's decision-making in the clinical workflow [6]. Examples of CDS functions are warning, alerts, and reminders on various aspects of medical decision-making [7-9].

These functions are closely related to quality of care, healthcare utilization, and costs. EMR systems have the function of health information exchanges, which can reduce healthcare utilization and costs [10]. Functions of CDS directly affect healthcare professionals' decision making [11]. They could help dentists assess patients' needs for orthodontic treatments [12]. According to several studies on the CDS of EMR systems, the CDS functionalities were closely associated with the prevention of medical errors, best practices, and the prevention of medical errors and inappropriate prescriptions [13-15]. CDS in EMR systems is expected to be considered more important in the near future [16].

These functions of CDS and digitalized clinical documents are considered important factors for EMR sophistication as well [17]. According to the EMR adoption model of the Healthcare Information and Management Systems Society (HIMSS), both CDS functionalities and the existence of clinical documents are crucial features in identifying the EMR sophistication level as stages 3 to 6, respectively [18]. The level of sophistication of an EMR system is associated with good clinical care [19]. Thus, we may need to know these functionalities and the level of documentation to figure out the level of EMR sophistication. There have been several studies on CDS functionalities and lists of clinical items, such as allergy lists and problem lists [1,20,21]. Nevertheless, there is a paucity of studies regarding how various CDS functionalities and clinical documents have been installed or structured in the EMR systems.

Therefore, the objective of this study was to investigate the current status of CDS functions and the digitalization of clinical documents in EMR systems as an exploratory study. EMR systems are becoming more important, and most healthcare organizations use EMR systems these days. EMR systems are expected to be rapidly deployed in the near future [22]. The results of this study may contribute to the widespread dispersion of EMR systems throughout the healthcare industry and to the standardization of various clinical documents in healthcare settings.

\section{Methods}

\section{Study Design}

This exploratory study focused on the current status of EMR systems. It had a cross-sectional design, and the unit of analysis was a healthcare organization, such as a general hospital or a small hospital. For this purpose, we conducted a nationwide survey on the specifications of EMR systems from July 25, 2018 to September 30, 2018 in Korea. The contents of the survey were the number of clinical documents, number of digitalized clinical documents, data store format, EMR system installation (development) type, possibility of data extraction, existence of CDSS, existence of 36 digitalized clinical documents in the EMR system, and types of clinical documents coming from outside of hospitals. For clinical documents, this study focused on 36 pre-chosen clinical documents considered important for the health insurance claim review and assessment by the Health Insurance Review \& Assessment Service (HIRA).

Respondents of the survey were mainly medical recorders. However, if they were not available, the study allowed staff members in the Department of Information and Communication Technology (ICT) and administrative staff members dealing with health insurance claims in the billing department to participate in the survey. The survey was conducted online. The online survey was developed and linked with the homepage website of the Korean Health Information Management Association (KHIMA). KHIMA sent a message to its members through mobile and e-mail message to inform them of the survey and encouraged them to participate. In addition, HIRA advertised its study through its official homepages and its official survey letter to each study target hospital through e-mail and encouraged them to participate through the survey link on KHIMA's homepage. HIRA also directly attached the survey questionnaire to an e-mail with the official survey letters. 
The study population was 344 general hospitals including 43 tertiary hospitals and 1,462 small hospitals. This study set all general hospitals and $21 \%$ of the small hospitals as the study sample. One hundred hospitals participated in the survey among a total of 344 general hospitals, which showed the response rate of $29.1 \%$. However, this study excluded two hospitals from the analysis because they did not have EMR systems. Of the 304 small hospitals sampled, 35 participated in the survey (response rate $=11.5 \%$ ). One small hospital was excluded from the analysis because it did not have an EMR system. We also used secondary administrative data from the national health insurance programs, such as foundation type, location, and number of beds in responding hospitals. This manuscript was an outcome of part of a project conducted by HIRA titled "Developing a National Strategic Plan for Health Insurance Claims Review and Assessment with Healthcare Information Exchanges" completed on December 30, 2018. The study was approved by the Institutional Review Board of HIRA on February 5, 2018 (No. 2018-004-001).

\section{Outcome Variable}

This study had two main outcome variables: functional status and the digitalization of clinical documents in EMR systems. In addition to these two main outcome variables, we also identified several variables, such as the approximate number of clinical documents, overall digitalization of clinical documents, and clinical documents most frequently observed at hosptials coming from outside of hosptials. These variables were analyzed with two outcome variables. According to two previous studies [1,21], hospitals are more likely to have comprehensive EMR systems as their size increases. Therefore, we considered these variables for two types of hospitals: general hospitals and small hospitals. In Korea, general hospitals are facilities having either 100 to 299 beds and at least seven medical specialties or more than 300 beds and nine specialties. Hospitals (hereafter, small hospitals) are those having more than 30 beds but do not meet the two criteria above. Finally, regarding the CDS functions of EMR systems, we selected six lists based on previous studies: warnings on drug dosage, reminders on the schedule of examinations and tests, order set of prescriptions, clinical practice guidelines, creating clinical documents or documentation templates, and drug utilization review (DUR) functions within EMR systems [7,9,21].

\section{Statistical Analysis}

We first examined the general characteristics of study subjects using descriptive statistics between two hospital types: general hospitals and small hospitals. When the frequency of observation was less than 5 , we set forth the $p$-value of Fisher exact test. If there were more than two-by-two metrics of the contingency table, then we cited the $p$-value of the Mantel-Haenszel chi-square test. For numeric variables, we used a group t-test. With regard to variables measuring the numbers of clinical documents and digitalized clinical documents, there were 5 missing values, 4 for general hospitals and one for a small hospital. We replaced those missing values with the average number of each hospital group. Finally, the SAS version 9.1 was used for the data analysis.

Table 1. General characteristics of responding hospitals

\begin{tabular}{|c|c|c|c|}
\hline Variable & $\begin{array}{l}\text { General hospitals } \\
\qquad(\mathrm{n}=98)\end{array}$ & $\begin{array}{l}\text { Small hospitals } \\
\qquad(n=34)\end{array}$ & $p$-value \\
\hline Foundation & & & $<0.001$ \\
\hline Private hospitals & $14(14.3)$ & $26(76.5)$ & \\
\hline Non-profit or public hospitals & $84(85.7)$ & $8(23.5)$ & \\
\hline Location & & & 0.236 \\
\hline Seven mega-metro cities including Seoul & $49(50.0)$ & $21(61.8)$ & \\
\hline The rest of the cities & $49(50.0)$ & $13(38.2)$ & \\
\hline Average number of beds & $626.9 \pm 456.7$ & $154.8 \pm 65.6$ & $<0.001$ \\
\hline Respondents & & & $<0.001$ \\
\hline Medical recorders & $83(84.7)$ & $9(26.5)$ & \\
\hline Staff members of ICT or billing department & $15(15.3)^{\mathrm{a}}$ & $25(73.5)^{b}$ & \\
\hline
\end{tabular}

Values are presented as number (\%) or mean \pm standard deviation.

aAll were staff members of ICT department, ${ }^{b} 13$ (38.2) in billing department. 


\section{Results}

The characteristics of the responding hospitals are presented in Table 1. A total of 98 general hospitals and 34 small hospitals participated in the survey. The percentages of private hospitals were $14.3 \%$ for general hospitals and $76.5 \%$ for small hospitals. The percentages of general hospitals and small hospitals located in 7 mega-metro cities including Seoul were $50.0 \%$ and $61.8 \%$, respectively. The average numbers of beds in general hospitals and small hospitals were 626 and 155, respectively. While the respondents of general hospitals were mostly medical recorders, the respondents of hospitals were both administrative staff members working in the billing department and specialized staff working on computer systems.

Table 2 shows the general characteristics of EMR product specifications. The average numbers of clinical documents of general hospitals and hospitals were 1,022 and 159, respectively. The percentage of clinical documents digitalized in EMR systems was approximately $80 \%$ in both general hospitals and hospitals. Almost 34\% and 38\% of general hospitals and small hospitals said that they had full EMR systems and did not have any paper form of clinical document. Only $10 \%$ of EMR systems were provided by vendors in general

Table 2. Characteristics of EMR product specifications

\begin{tabular}{|c|c|c|c|}
\hline Category & $\begin{array}{l}\text { General hospitals } \\
\qquad(\mathrm{n}=98)\end{array}$ & $\begin{array}{l}\text { Small hospitals } \\
\qquad(\mathrm{n}=34)\end{array}$ & $p$-value \\
\hline Average number of clinical documents before EMR installation & 1,022 & 159 & $<0.001$ \\
\hline Average number of clinical documents digitalized & 858 & 118 & $<0.001$ \\
\hline$\%$ of clinical documents digitalized in each hospital & 79.8 & 79.1 & 0.898 \\
\hline Level of clinical documents digitalized (\%) & & & 0.759 \\
\hline 100 & $33(33.7)$ & $13(38.2)$ & \\
\hline $90-99$ & $21(21.4)$ & $5(14.7)$ & \\
\hline $70-89$ & $16(16.3)$ & $8(23.5)$ & \\
\hline$\leq 69$ & $28(28.6)$ & $8(23.5)$ & \\
\hline EMR installation types & & & $<0.001$ \\
\hline Inside or outside development & $88(89.8)$ & $21(61.8)$ & \\
\hline Provided by vendors & $10(10.2)$ & $13(38.2)$ & \\
\hline Data storage format & & & 0.854 \\
\hline XML/text EMR or both formats (a) & $25(25.5)$ & $8(23.5)$ & \\
\hline Image EMR format (b) & $5(5.1)$ & $2(5.9)$ & \\
\hline The other format: (a) and (b) & $68(69.4)$ & $24(70.6)$ & \\
\hline
\end{tabular}

Values are presented as number (\%).

EMR: Electronic Medical Record.

Table 3. Clinical decision supports functions of EMR systems (unit: \%)

\begin{tabular}{|c|c|c|c|c|}
\hline No. & Category $^{a}$ & $\begin{array}{l}\text { General hospitals } \\
\qquad(\mathrm{n}=98)\end{array}$ & $\begin{array}{l}\text { Small hospitals } \\
\qquad(\mathrm{n}=34)\end{array}$ & $p$-value \\
\hline 1 & Warnings on drug dosage & 77.6 & 50.0 & 0.002 \\
\hline 2 & Reminders on the schedule of exam \& test & 70.4 & 35.3 & $<0.001$ \\
\hline 3 & Order set of prescriptions & 88.8 & 82.4 & 0.335 \\
\hline 4 & Clinical practice guidelines & 43.9 & 17.7 & 0.006 \\
\hline 5 & Creating clinical documents (documentation templates) & 67.4 & 29.4 & $<0.001$ \\
\hline 6 & Drug utilization review & 56.1 & 47.1 & 0.361 \\
\hline
\end{tabular}

EMR: Electronic Medical Record.

${ }^{a}$ Binary measure: having or not having those functionalities. 
Table 4. Precentage (\%) of clinical documents digitialized and observed in EMR systems

\begin{tabular}{|c|c|c|c|c|}
\hline Category & Clinical documents & $\begin{array}{c}\text { General hospitals } \\
(n=98)\end{array}$ & $\begin{array}{c}\text { Small hospitals } \\
\quad(n=34)\end{array}$ & $p$-value \\
\hline \multirow[t]{2}{*}{ Outpatients } & Outpatient medical records & 89.8 & 91.2 & 1.000 \\
\hline & Outpatient progress notes & 87.8 & 88.2 & 1.000 \\
\hline \multirow[t]{13}{*}{ Inpatients } & Inpatient medical records & 90.8 & 79.4 & 0.079 \\
\hline & Inpatient progress notes & 92.9 & 91.2 & 0.717 \\
\hline & Physician’s order notes & 84.7 & 76.5 & 0.276 \\
\hline & Admission nursing assessments & 86.7 & 79.4 & 0.305 \\
\hline & Nursing notes & 88.8 & 88.2 & 1.000 \\
\hline & Physician's progress notes & 67.5 & 41.2 & 0.007 \\
\hline & Discharge summary notes & 91.8 & 70.6 & 0.002 \\
\hline & Intensive care unit flowsheets & 67.4 & 14.7 & $<0.001$ \\
\hline & Operation records & 92.9 & 82.4 & 0.077 \\
\hline & Operation nursing care records & 86.7 & 55.9 & 0.000 \\
\hline & Anesthesia records & 78.6 & 73.5 & 0.546 \\
\hline & Recovery records & 79.6 & 38.2 & $<0.001$ \\
\hline & On/off duty notes & 48.0 & 11.8 & 0.000 \\
\hline \multirow[t]{3}{*}{ Laboratory } & Diagnostic test reports/results & 85.7 & 88.2 & 1.000 \\
\hline & Laboratory reports/results & 85.7 & 82.4 & 0.638 \\
\hline & Image (radiology) reports & 83.7 & 85.3 & 0.824 \\
\hline \multirow[t]{18}{*}{ Common areas } & Medication administration records & 81.6 & 82.4 & 0.925 \\
\hline & Prescription forms & 69.4 & 82.4 & 0.144 \\
\hline & Procedure notes & 73.5 & 55.9 & 0.056 \\
\hline & Emergency department records & 89.8 & 35.3 & $<0.001$ \\
\hline & Emergency department nursing notes & 87.8 & 29.4 & $<0.001$ \\
\hline & Consultation notes & 87.8 & 88.2 & 1.000 \\
\hline & Physician’s notes & 78.6 & 91.2 & 0.125 \\
\hline & Hemodialysis records & 70.4 & 17.7 & $<0.001$ \\
\hline & Physical therapy notes & 50.0 & 38.2 & 0.236 \\
\hline & Psychiatric therapy notes & 44.9 & 11.8 & 0.000 \\
\hline & Fall/decubitus prevention care notes & 82.7 & 64.7 & 0.029 \\
\hline & Patient education notes & 50.0 & 20.6 & 0.003 \\
\hline & Transfer in notes & 75.5 & 41.2 & 0.001 \\
\hline & Transfer out notes & 78.6 & 44.1 & 0.000 \\
\hline & Check sheets on quality improvement for DRG & 45.9 & 20.6 & 0.009 \\
\hline & IVF procedure reports & 21.4 & 0.0 & - \\
\hline & Rehabilitation evaluation reports & 46.9 & 14.7 & 0.001 \\
\hline & Medical expenses statements & 45.9 & 82.4 & 0.000 \\
\hline
\end{tabular}

EMR: Electronic Medical Record, DRG: Diagnosis-Related Group, IVF: in vitro fertilization.

hospitals, whereas $38.2 \%$ of EMR systems were provided by vendors in small hospitals. Approximately $26 \%$ and $24 \%$ of data were stored with XML, text, or both in general hospitals and hospitals, respectively.
Table 3 shows the CDS functionality status of EMR systems. The top CDS function of EMR systems in general and small hospitals was order set of prescriptions, respectively. For CDS functions such as warnings on drug dosage, re- 
minders on the schedule of exams and tests, and clinical practice guidelines, their proportion for general hospitals was statistically significantly higher than for small hospitals.

Table 4 presents the percentages of clinical documents located in the EMR systems of general hospitals. The highest percentage was the inpatient progress notes $(92.9 \%)$ and operation records (92.9\%), followed by discharge summary notes (91.8\%) among 36 clinical documents in EMR systems.

Table 5. Percentage (\%) of clinical documents most frequently observed at hosptials coming from outside of hosptials

\begin{tabular}{|c|c|c|c|c|}
\hline Category & Clinical documents & $\begin{array}{l}\text { General hospitals } \\
\qquad(\mathrm{n}=98)\end{array}$ & $\begin{array}{l}\text { Small hospitals } \\
\qquad(\mathrm{n}=34)\end{array}$ & $p$-value \\
\hline \multirow[t]{2}{*}{ Outpatients } & Outpatient medical records & 54.1 & 32.4 & 0.029 \\
\hline & Outpatient progress notes & 46.9 & 26.5 & 0.037 \\
\hline \multirow[t]{13}{*}{ Inpatients } & Inpatient medical records & 52.0 & 29.4 & 0.023 \\
\hline & Inpatient progress notes & 56.1 & 29.4 & 0.007 \\
\hline & Physician’s order notes & 32.7 & 11.8 & 0.024 \\
\hline & Admission nursing assessments & 21.4 & 11.8 & 0.310 \\
\hline & Nursing notes & 22.5 & 11.8 & 0.217 \\
\hline & Physician's progress notes & 15.3 & 2.9 & 0.069 \\
\hline & Discharge summary notes & 60.2 & 20.6 & $<0.001$ \\
\hline & Intensive care unit flowsheets & 9.2 & 5.9 & 0.728 \\
\hline & Operation records & 59.2 & 29.4 & 0.003 \\
\hline & Operation nursing care records & 7.1 & 0.0 & - \\
\hline & Anesthesia records & 8.2 & 0.0 & - \\
\hline & Recovery records & 9.2 & 0.0 & - \\
\hline & On/off duty notes & 5.1 & 0.0 & - \\
\hline \multirow[t]{3}{*}{ Laboratory } & Diagnostic test reports/results & 75.5 & 50.0 & 0.006 \\
\hline & Laboratory reports/results & 69.4 & 44.1 & 0.009 \\
\hline & Image (radiology) reports & 72.5 & 50.0 & 0.017 \\
\hline \multirow[t]{18}{*}{ Common areas } & Medication administration records & 45.9 & 38.2 & 0.437 \\
\hline & Prescription forms & 48.0 & 35.3 & 0.201 \\
\hline & Procedure notes & 22.5 & 5.9 & 0.038 \\
\hline & Emergency department record & 41.8 & 17.7 & 0.011 \\
\hline & Emergency department nursing note & 15.3 & 2.9 & 0.069 \\
\hline & Consultation note & 18.4 & 11.8 & 0.437 \\
\hline & Physician's note & 62.2 & 58.8 & 0.724 \\
\hline & Hemodialysis record & 12.2 & 8.8 & 0.759 \\
\hline & Physical therapy note & 5.1 & 5.9 & 1.000 \\
\hline & Psychiatric therapy note & 7.1 & 2.9 & 0.679 \\
\hline & Fall/decubitus prevention care note & 2.0 & 8.8 & 0.108 \\
\hline & Patient education note & 1.0 & 2.9 & 0.450 \\
\hline & Transfer in note & 11.1 & 5.9 & 0.513 \\
\hline & Transfer out note & 11.2 & 11.8 & 1.000 \\
\hline & Checking sheet on quality improvement for KDRG & 2.0 & 0.0 & - \\
\hline & IVF procedure report & 2.0 & 0.0 & - \\
\hline & Rehabilitation evaluation report & 4.1 & 0.0 & - \\
\hline & Medical expenses statement & 7.1 & 11.8 & 0.473 \\
\hline
\end{tabular}

EMR: Electronic Medical Record, KDRG: Korean Diagnosis-Related Group, IVF: in vitro fertilization. 
For small hospitals, the highest percentage (91.2\%) was equally observed for outpatient medical records, the inpatient progress notes, and physician's notes among 36 clinical documents in EMR systems. The proportion of digitalized clinical documents in general hospitals was generally higher than in small hospitals.

Table 5 presents the percentages of most frequent types of clinical documents from transferring facilities observed at participating hospitals. For general hospitals, clinical documents frequently observed from transferring facilities were image reports (72.5\%) followed by diagnostic test reports/ results $(71.5 \%)$ and laboratory reports/results (69.4\%). For small hospitals, the highest percentage was observed for physician's notes (58.8\%), followed by diagnostic test reports/ results (50.0\%) and image reports (50.0\%)

\section{Discussion}

This study produced several interesting exploratory findings. For EMR product specifications, this study found that almost $80 \%$ of clinical documents were digitalized in both general hospitals and small hospitals. The percentages of general hospitals and small hospitals with $100 \%$ paperless medical charts were $33.7 \%$ and $38.2 \%$, respectively. The percentages of EMR systems storing information in image format were less than 6\% for both hospital groups. Regarding CDS functions, the EMR systems of general hospitals are more likely to have functions of warnings on drug dosage, reminders on the schedule of examinations and tests, and clinical practice guidelines in comparison to those of small hospitals, which was statistically significant. Regarding the lists of digitalized clinical documents, almost 93\% of EMR systems in general hospitals have inpatient progress notes, operation records, and discharge summary notes digitalized. The proportion of digitalized documents of EMR systems in small hospitals was much lower than that in general hospitals for each clinical document category. Finally, for the clinical documents coming from outside hospitals, the clinical documents most frequently observed at general hospitals were image reports (72.5\%) followed by diagnostic test results (71.5\%), and laboratory test results $(69.4 \%)$, which was statistically higher than those of small hospitals.

The study results from EMR product specifications that only $35 \%$ of hospitals have $100 \%$ paperless clinical documents in their EMR systems suggests that there is still a long way to go towards the development of sophisticated EMR systems. However, the fact that only $6 \%$ of EMR systems have image data storage format is a good sign for HIE be- cause documents stored in image format make HIE difficult. Regarding the adoption rate of CDS functions of EMR systems, we found higher rates than a previous study conducted in Korea [21]. While the previous study targeting general hospitals found CDS adoption rates such as drug-dose support $(24.3 \%)$, laboratory interaction alerts $(28.7 \%)$, clinical guidelines (33.9\%), clinical reminders (38.0\%), and drugdrug interaction alerts $(65.0 \%)$, we found the following rates: warnings on drug dosage $(77.6 \%$ in general hospitals, $50.0 \%$ in small hospitals), reminders on the schedule of examinations and tests (70.4\%, 35.3\%), clinical guidelines (43.9\%, 17.7\%), and DUR (56.1\%, 47.1\%). Regarding the digitalization of clinical documents, our results show that there is a gap between a full set of digitalized clinical documents and perfect EMR systems. The highest rate of adoption was observed for inpatient progress notes (92\%), which means the rest of the EMR systems (8\%) might not handle this type of clinical document. Assuming that the adoption rate of any kind of EMR systems in Korea approximately reaches 96\% [3], government healthcare ICT policies should focus on EMR sophistication from now on. Regarding the most frequently observed clinical documents coming from outside of hosptials, the study results showed that clinical documents on various test results or image reports have high frequency of observation. Our results are in line with those of a previous study in which an HIE group exchanged a higher number of information items, such as clinical laboratory tests and diagnostic imaging results, in comparison to a non-HIE group [10].

There were several limitations of this study. First, there was a low response rate (11.5\%) from small hospitals in the study population. A low response rate, generally speaking, reduces statistical power, which affects study validation. The likelihood of having a department of medical records is low in small hospitals, which could account for the low participation rate. Second, this study was exploratory; hence, it did not include deep statistical analysis. Further rigorous analysis should be conducted with diverse perspectives. Third, we targeted a group of 36 clinical documents which were chosen by HIRA. We may have missed important clinical documents that were not chosen by HIRA. Finally, interpretation of our results may be limited to Korea. This study does not have any comparative studies because there was no previous reference, especially focusing on the diversity of clinical documents.

In summary, this exploratory study investigated the functionalities of CDS and the types of clinical documents in EMR systems. Our results showed that most EMR systems 
have various CDS functionalities and there was a huge variation regarding the lists of clinical documents digitalized. In particular, the difference between general hospitals and small hospitals was large. These result suggest that EMR systems in Korea need to be developed with a more sophisticated design reflecting a wider variety of clinical documents.

This study indirectly implies that the digital environment of EMR system needs to enhanced through the adoption of sophisticated EMR systems, which means that there is much room for improvement of EMR system. EMR systems are deeply connected and rooted in hospitals' organizational structure and ICT infrastructure [23]. We must understand the various features of EMR systems to achieve successful implementation of EMR systems [24]. The results of this study could be used for the standardization of CDS function and clinical documents. Moreover, these results make a meaningful contribution to the development of EMR systems by providing the overall current status of CDS functionalities and the level of digitalization of various clinical documents.

\section{Conflict of Interest}

Young-Taek Park is an editor of Healthcare Informatics Research; however, he did not involve in the peer reviewer selection, evaluation, and decision process of this article. Otherwise, no potential conflict of interest relevant to this article was reported.

\section{Acknowledgments}

We deeply thank to the President, Executive Director, and its members of Korean Health Information Management Association (KHIMA) for their assistance and help to collect the survey from their members, medical recorders, of the hospitals.

\section{ORCID}

Young-Taek Park (http://orcid.org/0000-0002-7574-4165)

Yeon Sook Kim (http://orcid.org/0000-0002-7368-7312)

Byoung-Kee Yi (http://orcid.org/0000-0002-7699-9629)

Sang Mi Kim (http://orcid.org/0000-0002-6657-3624)

\section{References}

1. Jha AK, DesRoches CM, Campbell EG, Donelan K, Rao SR, Ferris TG, et al. Use of electronic health records in
U.S. hospitals. N Engl J Med 2009;360(16):1628-38.

2. Holroyd-Leduc JM, Lorenzetti D, Straus SE, Sykes L, Quan $\mathrm{H}$. The impact of the electronic medical record on structure, process, and outcomes within primary care: a systematic review of the evidence. J Am Med Inform Assoc 2011;18(6):732-7.

3. Park YT, Han D. Current status of electronic medical record systems in hospitals and clinics in Korea. Healthc Inform Res 2017;23(3):189-98.

4. Garrett P, Seidman J. EMR vs EHR - what is the difference? [Internet]. Washington (DC): The Office of the National Coordinator for Health Information Technology; c2019 [cited at 2019 Apr 15]. Available from: https://www.healthit.gov/buzz-blog/electronic-healthand-medical-records/emr-vs-ehr-difference.

5. Shachak A, Reis S. The impact of electronic medical records on patient-doctor communication during consultation: a narrative literature review. J Eval Clin Pract 2009;15(4):641-9.

6. The Office of the National Coordinator for Health Information Technology (ONC). Clinical decision support [Internet]. Washington (DC): ONC; c2019 [cited at 2019 Apr 15]. Available from: https://www.healthit.gov/topic/ safety/clinical-decision-support.

7. Osheroff JA, Teich JM, Middleton B, Steen EB, Wright A, Detmer DE. A roadmap for national action on clinical decision support. J Am Med Inform Assoc 2007;14(2):141-5.

8. Middleton B, Bloomrosen M, Dente MA, Hashmat B, Koppel R, Overhage JM, et al. Enhancing patient safety and quality of care by improving the usability of electronic health record systems: recommendations from AMIA. J Am Med Inform Assoc 2013;20(e1):e2-8.

9. Keenan GM, Lopez KD, Yao Y, Sousa VE, Stifter J, Febretti A, et al. Toward meaningful care plan clinical decision support: feasibility and effects of a simulated pilot study. Nurs Res 2017;66(5):388-98.

10. Park H, Lee SI, Hwang H, Kim Y, Heo EY, Kim JW, et al. Can a health information exchange save healthcare costs? Evidence from a pilot program in South Korea. Int J Med Inform 2015;84(9):658-66.

11. Khairat S, Marc D, Crosby W, Al Sanousi A. Reasons for physicians not adopting clinical decision support systems: critical analysis. JMIR Med Inform 2018;6(2):e24.

12. Thanathornwong B. Bayesian-based decision support system for assessing the needs for orthodontic treatment. Healthc Inform Res 2018;24(1):22-8.

13. Jao CS, Hier DB. Clinical decision support systems: an 
effective pathway to reduce medical errors and improve patient safety. In: Jao C, editor. Decision support systems. London: IntechOpen; 2010.

14. Scott IA, Pillans PI, Barras M, Morris C. Using EMR-enabled computerized decision support systems to reduce prescribing of potentially inappropriate medications: a narrative review. Ther Adv Drug Saf 2018;9(9):559-73.

15. Adler-Milstein J, Holmgren AJ, Kralovec P, Worzala C, Searcy T, Patel V. Electronic health record adoption in US hospitals: the emergence of a digital "advanced use" divide. J Am Med Inform Assoc 2017;24(6):1142-8.

16. Berner ES, La Lande T. Clinical decision support systems: impacting the future of clinical decision making. In Ball MJ, Weaver CA, Kiel JM, editors. Healthcare information management systems. New York (NY): Springer; 2004. p. 463-77.

17. Institute of Medicine (US) Committee on Data Standards for Patient Safety. Key capabilities of an electronic health record system: letter report. Washington (DC): National Academies Press; 2003.

18. Healthcare Information and Management Systems Society (HIMSS). EMR sophistication correlates to hospital quality data [Internet]. Chicago (IL): HIMSS; c2006 [cited at 2019 Apr 15]. Available from: https://www. himss.eu/content/himss-analytics-emr-sophisticationcorrelates-hospital-quality-data.
19. Tundia NL, Kelton CM, Cavanaugh TM, Guo JJ, Hanseman DJ, Heaton PC. The effect of electronic medical record system sophistication on preventive healthcare for women. J Am Med Inform Assoc 2013;20(2):268-76.

20. Adler-Milstein J, DesRoches CM, Kralovec P, Foster G, Worzala C, Charles D, et al. Electronic health record adoption in US hospitals: progress continues, but challenges persist. Health Aff (Millwood) 2015;34(12):217480.

21. Kim YG, Jung K, Park YT, Shin D, Cho SY, Yoon D, et al. Rate of electronic health record adoption in South Korea: a nation-wide survey. Int J Med Inform 2017;101:100-7.

22. Kharrazi H, Gonzalez CP, Lowe KB, Huerta TR, Ford EW. Forecasting the maturation of electronic health record functions among US hospitals: retrospective analysis and predictive model. J Med Internet Res 2018;20(8):e10458.

23. Lee YT, Park YT, Park JS, Yi BK. Association between electronic medical record system adoption and healthcare information technology infrastructure. Healthc Inform Res 2018;24(4):327-34.

24. Steiner BA. (2009). Electronic medical record implementation in nursing practice: a literature review of the factors of success [dissertation]. Bozeman (MT): College of Nursing, Montana State University; 2009. 